\title{
La producción de los espacios de la ciudad global: planificación estratégica, megaproyectos y las "necesidades" de mercado inmobiliarío ${ }^{1}$
}

\author{
Christof Parnreiter ${ }^{2}$
}

\begin{abstract}
RESUMEN
El texto analiza la relación entre la formación de ciudad global, el auge en la economía de bienes raíces y los cambios en la planificación urbana en la Ciudad de México. Se demuestra que la afluencia masiva de capital (extranjero) en la economía de bienes raíces ha alimentado un auge de la construcción, mientras el crecimiento del sector de servicios al productor ha estimulado la demanda de espacios de oficina. La producción de estos espacios de ciudad global requería, sin embargo, cambios importantes en la planificación urbana. A pesar de que la ciudad está gobernada desde hace casi 20 años por gobiernos de izquierda, se han implementado instrumentos de la planificación estratégica con el doble fin de hacer la planificación más rápida, flexible y específica de lugar y de centralizar la toma de decisiones. Tanto circuitos globales de conocimiento como presiones locales en la economía de bienes raíces son los principales mecanismos que han inducido los cambios en la política urbana en la Ciudad de México.
\end{abstract}

Palabras clave: Ciudad global, planificación estratégica, megaproyectos, economía de bienes raíces, Ciudad de México

\section{ABSTRACT}

This paper analyses the relation between global city formation, the real estate boom and shifts in urban planning in Mexico City. It shows that the massive influx of (foreign) capital to the real estate sector nourished a construction boom, while the growth of the producer service sector has spurred demand for office spaces. The production of these global city spaces required, however, changes in urban planning. Left-wing governments notwithstanding, instruments of strategic planning have been introduced with the dual goal of make planning faster, more flexible and site-specific, and on the other hand of centralizing decision-making. Both global circuits of knowledge and local pressures on the real estate economy have been the main mechanisms that have induced the changes in urban politics in Mexico City.

Key words: Global city, strategic planning, megaprojects, real estate economy, Mexico City

Artículo recibido el 28 de julio de 2015, aceptado el 9 de diciembre de 2015 y corregido el 8 de abril de 2016.

\footnotetext{
2 Departamento de Geografía, Universidad de Hamburgo (Alemania).

E-mail: christof.parnreiter@uni-hamburg.de
} 
Como muchas otras ciudades, la Ciudad de México ha visto en las últimas dos décadas la producción de nuevos centros dentro de la ciudad, que proporcionan la infraestructura física para un nuevo tipo de centralidad económica. Esta es, según Sassen (1991) una centralidad dispersa geográficamente, que toma la forma de una red transfronteriza de ciudades globales. Desde una perspectiva del "Sur global" cabe destacar que esta red atraviesa la tradicional brecha de poder NorteSur por la integración de las ciudades en el "primer" como en el "tercer" mundo (para un reporte empírico véase Taylor et al., 2011, para América Latina véase Parnreiter, 2015). Entrar a formar parte de esta red mundial de centralidad económica provoca profundas transformaciones urbanas en las ciudades, de las cuales la más sobresaliente es un fuerte crecimiento del sector de servicios. Este crecimiento tiende a profundizar las desigualdades existentes de las economías y las sociedades urbanas, $y$ ha causado masivas intervenciones en el entorno construido. La formación de las ciudades globales ha provocado "una nueva arquitectura de la centralidad que representa y aloja nuevas formas de poder económico, es decir, el hiperespacio de negocios internacionales; testificados por las torres corporativas, hoteles corporativos, $y$ aeropuertos de clase mundial que han constituido una nueva geografía del entorno construido de centralidad" (Sassen, 1996: 208, traducción del autor).

En las ciudades de los países más pobres la reestructuración de la forma urbana es especialmente relevante, ya que por mucho tiempo no contaban con actividades económicas que son centrales a escala mundial. Sin embargo, debido a todas las instituciones financieras, firmas legales y contables y consultorías empresariales que han seguido a sus clientes a América Latina, las ciudades africanas o asiáticas, requieren espacios de oficina de alta calidad, y porque el inventarío existente en estas ciudades no podía ni cualitativamente ni cuantitativamente satisfacer la demanda, surgió la necesidad de adaptar la estructura física de las ciudades del "Sur global" a las necesidades de las empresas transnacionales. Es de allí que surge la producción de nuevos centros económicos dentro de las ciudades.
Después de introducir el marco teórico, en este trabajo se revisa brevemente este desarrollo en la Ciudad de México, para luego explicarlo mediante dos fuerzas interrelacionadas que impulsan este proceso: Mientras que en el lado de la oferta, la afluencia masiva de capital (extranjero) en la economía de bienes raíces ha alimentado un auge de la construcción sin precedentes, en el lado de la demanda el crecimiento del sector de servicios en el proceso de formación de ciudades globales ha estimulado principalmente la demanda de espacios de oficina. Además, voy a esbozar algunos cambios importantes en la planificación urbana de la Ciudad de México, para luego demostrar que han sido fundamentales para la producción de los nuevos centros económicos de la ciudad global. Por último, voy a hablar de los circuitos globales de conocimiento y presiones locales en la economía de bienes raíces como principales mecanismos que han inducido los cambios en la política urbana en la Ciudad de México.

La investigación que subyace este texto ha sido llevada a cabo utilizando un enfoque de método mixto. Para las dos primeras secciones he recogido y analizado los datos cuantitativos sobre la economía de bienes raíces, de la inversión extranjera directa, del desempeño del sector de servicios de la Ciudad de México y de los patrones de localización de las empresas más grandes de México. Las fuentes de datos fueron: a) los informes trimestrales y anuales de las empresas de bienes raíces (a saber, CB Richard Ellis, Colliers International, Cushman \& Wakefield y Jones Lang LaSalle); b) los bancos de datos del INEGI y de la Secretaría de Economía; c) el banco de datos de CNN / Expansión sobre las 500 empresas más grandes de México; d) la literatura secundaria. En el tercer y cuarto capítulos sobre planificación urbana en la Ciudad de México he usado un enfoque cualitativo. La información se obtuvo, por un lado, a través de un análisis de las leyes, decretos, comunicados oficiales y otras publicaciones del gobierno de la ciudad, de la cobertura de los medios de desarrollos inmobiliaríos, de sitios web y otras publicaciones de las empresas que participan en la construcción y arrendamiento de torres de oficinas, de información distribuida por ONG urbanas y los partidos políticos en la Ciudad de México. Por otro lado, llevé a cabo en- 
trevistas semiestructuradas a expertos como arquitectos, urbanistas y ex funcionarios de la SEDUVI, todos los cuales se han visto involucrados en la planificación de la Ciudad de México. Las entrevistas fueron en torno a tres grandes áreas temáticas. En primer lugar, se pidió a los entrevistados describir y evaluar los principales cambios en las políticas y procedimientos de planificación, así como sus resultados (lo que incluye, por ejemplo, ejemplificaciones detalladas de instrumentos específicos). En segundo lugar, los expertos deberían rastrear influencias en las políticas de Planificación en la Ciudad de México con un enfoque específico en los actores y sus geografías. En tercer lugar, se pidió a los encuestados que dieran información sobre y evaluar las relaciones entre el Departamento de planificación de la ciudad y el sector privado, a saber, los desarrolladores y otras empresas de bienes raíces.

\section{Mercado inmobiliario, financiarización y urbanismo transnacional}

Conceptualmente, este trabajo se nutre de dos acercamientos teóricos. Primero, para comprender el auge del sector inmobiliarío tanto en la $\mathrm{Cd}$. de México como en muchas otras ciudades de América Latina y más allá, es conveniente recurrir a la conceptualización de David Harvey de la relación entre las crisis recurrentes del sistema capitalista y "la urbanización del capital" (Harvey, 1985). En esencia la teoría de Harvey intenta a desvelar "la relación entre la dinámica temporal de la acumulación de capital y la producción de nuevas configuraciones espaciales" (Harvey, 1985: 33; traducción del autor). Dice Harvey que en tiempos de acumulación desacelerada en el primer circuito del capital, o sea, en la producción (industrial) de mercancías, que se manifiesta en la caída de las tasas de ganancia, el capital está desplazado hacia un segundo circuito, el del capital fijo. En este contexto destacan las inversiones en el entorno construido como un proceso en que el capitalismo "crea un paisaje físico de carreteras, viviendas, fábricas, escuelas, tiendas, etcétera, en su propio imagen" (Harvey, 1985: XV, traducción del autor). Dicho de otra manera, en tiempos de una generalizada disminución de los retornos a la inversión, las inversiones inmobiliarias constituyen "una especie de última esperanza para encontrar usos productivos para la acumulación de capital rápida" (Harvey, 1985: 20, traducción del autor). Es por eso que la crisis del Fordismo -como la más reciente crisis de sobreacumulación- y la llamada globalización se caracterizan, entre otros, por procesos de desindustrialización en los centros de la economía global, una reubicación de capitales hacia la esfera financiera (la llamada financiarización), y una fusión de los mercados financieros e inmobiliaríos (Arrighi, 1994). Según el famoso concepto del spatial fix del capital de Harvey (Harvey, 2001), en este proceso el capital está fijado en el espacio -invertido en el paisaje urbano productivo del capitalismo está hecho inmóvil. Sin embargo, y visto desde la perspectiva del entorno construido, muchos de los inmuebles- término que ya señala que están atados al territorío tanto físicamente como jurídicamente -fueron movilizados en la medida en que son cada vez más utilizados como activos financieros. Mientras esta transformación en el significado de los inmuebles no está reducida al ámbito de los edificios de oficinas de primera clase (Aalbers, 2012, por un análisis de los mercados hipotecaríos en la crisis 'subprime'), las oficinas en las ciudades globales (como la Ciudad de México, ver Parnreiter, 2010) sí constituyen un campo excepcional por su creciente sector de servicios (Haila, 2000; Lizieri \& Pain, 2014). Las ciudades globales juegan un papel extraordinarío en la globalización del sector inmobiliarío (Stevenson et al., 2014), por lo cual el mercado inmobiliarío mundial está ubicado principalmente en ellas (Sassen, 1991; Fainstein, 2001). Lizieri (2012) ha señalado que en ciudades globales, tales como Londres, la propiedad global de oficinas ha aumentado drásticamente desde la década de 1980, principalmente, porque la distinción entre el financiamiento del desarrollo inmobiliario, la propiedad de bienes raíces como una inversión y la ocupación de la propiedad se han desdibujado.

El segundo enfoque teórico que nutre este trabajo es el concepto del urbanismo transnacional (Smith, 2001; Krätke et al., 2012). Esta noción se refiere a actores urbanos -desde migrantes sobre políticos a los desarrolladores de bienes raíces- que no solamente 
operan transfronterizo, sino que carecen de una identidad nacional clara. La noción transnacionalidad se refiere por ende, al grado de intermediación entre el aquí y el allí. En consecuencia, "ciudades transnacionales" se teorizan como "creaciones humanas que mejor se entienden como sitios de agencia con múltiples centros, si no descentrada" (Smith, 2001: 70, traducción del autor). De acuerdo con tales razonamientos, Anthony King (2004: cap. 5) propone que la historia de la planificación urbana debe ser referida y se escribe como "historias de planificación transnacionales", mientras las ciudades deben entenderse "no en relación con una, sino dos o más sociedades" (King, 2004: 82). Un resultado de estos procesos es la aparición de la torre de oficinas "como un dispositivo de clasificación social" (Grubbauer, 2014, traducción del autor), la producción de la arquitectura también se ha convertido en un importante campo de la normalización transfronteriza.

Un caso actual de ello es la planificación estratégica, que hoy se aplica en todas las partes del mundo (Carmona, 2009; ONUHábitat, 2009). El punto importante aquí es, sin embargo, no solo el cambio de planes estratégicos en todo el mundo, sino la forma en que se logró y lo que ocurrió con la planificación estratégica en el proceso de difusión global. Mi argumento es que debido a la constelación específica en la que se organiza la circulación transfronteriza del conocimiento, la planificación estratégica se ha separado de sus orígenes nacionales y por lo tanto de las identidades "originales" que tenía. Hoy en día, los expertos, experiencias e ideas, que son a la vez anclado localmente y fluyendo a través de fronteras, son decisivos en la conformación de los numerosos planes estratégicos implementados. La planificación estratégica ha, por ende, acumulado una historia multicéntrica y un carácter transnacional. Sin embargo, eso no se debe equiparar con igualdad de participación en la elaboración de dichas políticas: La formación de una política urbana transnacional es un proceso desigual en el que solo las ideas específicas sobre el desarrollo urbano son elegidos para convertirse en un nuevo estándar global. Este estándar sirve para lo que Brenner y Teodoro (2002: 353, traducción del autor) han Ilamado la "imposición del liberalismo en todo el mundo", pero a la escala de la ciudad.

\section{Un nuevo centro económico en la Ciudad de México}

Desde finales de los años ochenta, la geografía corporativa $^{3}$ en la Ciudad de México se ha transformado profundamente. En 2010, el CDB tradicional, compuesto por el corredor Centro Histórico - Paseo de la Reforma, la zona de Polanco y Avenida Insurgentes, representó solo el $30 \%$ del volumen de las ventas de las 500 grandes empresas en México, mientras que el CDB nuevo, construido desde la década de 1980 del oeste hacia el centro (Lomas Altas, Bosque de Lomas y Santa Fe), ya hizo un 26\%. Esta descentralización ocurrió en el curso de un auge de la construcción desde la entrada en vigor del TLCAN. Entre 1997 y 2011 el inventarío total de espacio de oficinas creció 3,7 millones de metros cuadrados, duplicando a 7,1 millones. Más de 800.000 metros cuadrados estaban en construcción a finales de 2012. Además de su fuerte expansión, cambió el mercado inmobiliarío en dos aspectos destacables. En primer lugar, hay una marcada tendencia hacia una mejora. Recientemente la construcción suministra oficinas principalmente para el primer segmento del mercado ( $\mathrm{A}$ y $\mathrm{A}+$ ), que aumentó su participación en el inventarío total a dos tercios.

En segundo lugar, la geografía del espacio de oficina cambió considerablemente. Durante los primeros años del boom de la construcción, se agregaron nuevas oficinas principalmente en el CBD nuevo, que en 2007 abarcó un poco más de la mitad del inventarío. Santa Fe se destacó particularmente como nuevo centro económico de la Ciudad de México, con $16 \%$ de todos los espacios de inmobiliarío. Sin embargo, desde entonces se recuperó el CDB tradicional, debido principalmente a un auge de la construcción masiva a lo largo del Paseo de la Reforma. Por mucho tiempo uno de los bulevares principales de la ciudad, Reforma perdió importancia en la década de 1980 debido a la desaparición de la estratégica de sustitución de importaciones y los daños causados por el terremoto en 1985.

\footnotetext{
3 Siguiendo Grant \& Nijman (2002), utilicé el término 'geografía corporativa' para denotar la distribución especial de la oficinas de las empresas más grandes.
} 
Sin embargo, con la construcción de la Torre Mayor a finales de la década de 1990 comenzó una recuperación que actualmente está llevando a una reapropiación de los espacios de la centralidad tradicional. Mientras que entre 2001 y 2009, la construcción de varias torres de oficinas y de uso mixto han añadido unos 277.000 metros cuadrados de espacio de inmobiliarío en Reforma (Márquez López, 2012), proyectos actualmente en marcha suministrarán más de 180.000 metros cuadrados. Sin embargo, el espacio de oficina principal aún se concentra en el CDB nuevo (que representa el $61 \%$ de todo el inmobiliarío), con Santa Fe (26\%) siendo el más importante submercado.

\section{Inversión extranjera directa en bienes raíces y el crecimiento del sector de servicios}

En línea con el razonamiento teórico presentado anteríormente, la inversión transfronteriza inmobiliaria creció fuertemente hasta la crisis de 2007, para hacer el 47\% de todas las transacciones a nivel global (US\$ 357 bn.). Como consecuencia de la crisis, el volumen y el porcentaje cayeron a US\$112 bn. y el $41 \%$, respectivamente (2011) (JLL, 2008, 2012a).

La Ciudad de México es un destino favorecido por la inversión inmobiliaria global. La inversión extranjera directa en servicios inmobiliaríos ha crecido (aunque con fluctuaciones) significativamente, y ha aumentado su participación en todas las IED en el Distrito Federal (Secretaría de Economía, 2012). Aunque tanto el volumen de las inversiones transfronterizas y su participación en las transacciones de bienes raíces comerciales de la Ciudad de México han caído significativamente en el curso de la crisis, entre 2007 y 2012 el capital extranjero representó el 59\% de todas las transacciones (US\$ 5,2 bn.) (JLL, 2012 b). Los vínculos estructurales entre los sectores inmobiliarío y financiero, los cuales Harvey (1985) teoriza como un elemento estructural en la crisis de sobreacumulación, también se revelan en un informe del Grupo Financiero BBVA-Bancomer (BBVA, 2012). Entre 2000 y 2011, la participación del sector de la construcción de México en el volumen total del crédito ha crecido cuatro veces, hasta $21,3 \%$. La industria de la construcción también se destaca como el sector con la mayor participación de las empresas endeudadas $-53 \%$ de todas las compañías usan financiamiento externo, en comparación con un $30 \%$ en el resto de la economía-. Esto, sin embargo, parece ser rentable: inmobiliarias con créditos tienen ingresos significativamente mayores que las empresas contando con financiamiento interno.

La adhesión de México al TLCAN es un punto de inflexión crucial. Primero, la liberalización financiera empujó la movilidad del capital y abrió las puertas para la IED. Mientras en términos generales, las entradas promedios de IED aumentaron más del doble desde 1994, la liberalización también abrió por primera vez después de la Revolución Mexicana el sector inmobiliarío a actores y capitales extranjeros. En 2004, por ejemplo una legislación encargada de regular a los fondos de inversión inmobiliaria (REIT, por sus siglas en inglés) fue introducida. Segundo, y relacionado a la creciente IED, tanto la presencia como la importancia de empresas transnacionales produciendo en México se ha aumentado significativamente (Dussel Peters, 2000). Conjunto con la nueva política de la industrialización orientada a la exportación, eso no solo hizo crecer fuertemente a las exportaciones, sino también cambió radicalmente su composición: la estructura del comercio exteríor cambió de exportaciones de recursos naturales (hasta la década de 1980 el petróleo fue con mucho el bien exportado más importante) a la industria manufacturera, que hoy en día asciende aproximadamente a $80 \%$ de las exportaciones. Eso implica que la economía en México hoy en día está mucho más integrada en cadenas productivas que hace 20 años (Parnreiter, 2013), lo que explica un tercer aspecto por qué el TLCAN significa un punto de inflexión en el desarrollo de la producción de los espacios centrales en la Ciudad de México. Según la teoría de la ciudades globales (Sassen, 1991), más globalización económica implica una creciente demanda por servicios al productor $y$, por ende, una creciente presencia de empresas globales que brindan estos servicios (Taylor et al., 2011).

El punto clave en el contexto de este documento es que la afluencia de empresas 
extranjeras de ambas sectores -los de la industria manufacturera y de los servicios al productor- ha aumentado significativamente la demanda por oficinas de primera clase en la Ciudad de México. Hay muchos indicios de que el auge de la construcción también ha sido impulsado por la demanda. La tasa de desocupación descendió de 23\% en 1998 al $5 \%$ en 2007 . Después de un aumento inducido por la crisis, comenzó a caer otra vez para ascender al $10 \%$ a finales de 2012. Las actividades de venta y arrendamiento, también se recuperaron, así que para Jones Lang Lasalle (2012: 2) la Ciudad de México es un "star market". Cabe mencionar que la formación de la ciudad global ha sido un factor decisivo para esta creciente demanda de espacios de oficina, porque ha causado un fuerte crecimiento del sector de servicios al productor ${ }^{4}$. En 2008, este sector representó el $46.5 \%$ del total del valor añadido en el Distrito Federal y $17,7 \%$ de los empleados formalmente. Lo importante aquí es, en primer lugar, la fuerte centralización de servicios al productor en el Distrito Federal, que representa el $75,8 \%$ del valor total del sector en México y $44,5 \%$ de todos los empleos en este sector. En segundo lugar, el sector de servicios al productor está creciendo rápidamente ${ }^{5}$. En la última década, los servicios financieros e inmobiliaríos aumentaron su participación en el producto del Distrito Federal por $65,9 \%$ (de 21,4 a $35,5 \%$ ) y en el empleo urbano en un $47,7 \%$ (de 7,7 a 11,5\%) (INEGI, 1999, 2009). Este crecimiento ha sido empujado por la afluencia masiva de firmas globales. En 2010, de las 175 empresas globales en el sector de servicios al productor analizadas por GaWC (Globalization and World Cities Research Network), 89 tenía oficinas en la Ciudad de México (Taylor et al., 2013).

El fuerte crecimiento del sector de servicios al productor ha estimulado y cambiado

\footnotetext{
4 Servicios financieros y de seguros; los servicios inmobiliaríos y de alquiler de bienes muebles e intangibles; servicios profesionales, científicos y técnicos; dirección de corporativos y empresa.

5 Ya que el INEGI ha alterado la clasificación de los subsectores económicos, una comparación temporal solo es posible para los servicios financieros e inmobiliaríos. Sin embargo, estos dos ascienden a dos tercios del empleo y tres cuartos del valor agregado en el sector de los servicios al productor.
}

la demanda de espacio de oficina de tal modo que promovió la producción de un nuevo CBD. Es Ilamativo que hay, en primer lugar, una clara dimensión histórica a la diferenciación geográfica entre viejo y nuevo CBD. Las empresas que ya desempeñaron un papel importante durante la industrialización por sustitución de importaciones tienen en su mayoría su sede en el tradicional CBD, mientras que las empresas que se han instalado en México desde la entrada en vigor del TLCAN muestran una clara preferencia por el nuevo CBD. En segundo lugar, existe también una geografía específica a las ubicaciones de las sedes de las empresas extranjeras: Mientras el nuevo CBD cuenta con los $48 \%$ de las ventas de las empresas extranjeras que figuran en la lista de las empresas Top 500, el CBD tradicional tiene solo $27 \%$. En tercer lugar, hay una dimensión sectorial para el cambio geográfico hacia el nuevo $\mathrm{CBD}$, aunque esto no es evidente a primera vista. Empresas de servicios al productor se encuentran igualmente con frecuencia en el antiguo (43\% de las ventas de las empresas de servicio de productor Top 500) y en el nuevo CBD (42\%) (Expansión, varíos años). Sin embargo, el CDB tradicional concentra las instituciones financieras a nivel nacional que tienen como propiedad atender el mercado interno (por ejemplo, Infonavit, Fovissste o Nafin). Por otra parte, el Grupo Financiero BBVA-Bancomer, ahora capital español Banco mexicano y el mandamás en el mercado, por razones históricas todavía se encuentra en el CDB antiguo, se ha mudado a Paseo de la Reforma a su nueve torre de oficinas a finales de 2015. Si hablamos de la Ciudad de México como ciudad global hablamos, por ende, de algunas zonas bien delimitables espacialmente. Su producción física ha sido facilitada por la flexibilización, la especificación y la centralización de la planificación urbana en las últimas dos décadas.

\section{Flexibilización, especificación y centralización de la planificación urbana en la Ciudad de México}

Durante mucho tiempo, la planificación urbana en la Ciudad de México ha sido dependiente particularmente de la toma de decisiones de los políticos o funcionarios 
(Ward, 1998). En la década de 1970, sin embargo, en el apogeo de la industrialización por sustitución de importaciones, se tomaron medidas hacia una democratización de la planificación. De esta manera entró en vigor el Plan General de Desarrollo Urbano del Distrito Federal (1980). Sin embargo, la desaparición del modelo de sustitución de importaciones en la década de 1980 y el cambio a las políticas neoliberales a nivel nacional y también en la escala urbana implicó también el fin de los intentos por llevar a cabo un desarrollo planificado, democrático, e inclusivo (Pradilla Cobos, 2009). A pesar de la reforma política fundamental de los años noventa en el Distrito Federal que trajo alcaldes elegidos democráticamente, y aunque la izquierda política gobernó el Distrito Federal desde 1997, las políticas urbanas han sido de dos filos: Mientras se introdujo un número de programas sociales para los grupos más vulnerables, los gobiernos de la ciudad también continuaron favorecer megaproyectos para satisfacer la demanda de los actores en la ciudad global (Olivera, 2014). Como resume Garza (1999: $165 f$, traducción del autor), que se ha convertido en el hilo conductor de las políticas urbanas desde finales de 1980 es que "las necesidades del mercado de bienes raíces determinan los proyectos que se Ilevarán a cabo y el uso de la tierra requerida". Los gobiernos del Distrito Federal elegidos democráticamente continuaron y profundizaron las reformas en la planificación urbana, que son diseñados a facilitar la intervención del Estado en este rubro haciendo la planificación más flexible y específica del lugar, volviendo a centralizar la toma de decisiones en manos de la SEDUVI, el Departamento de planificación de la ciudad, y del Jefe de Gobierno.

Para lograr este objetivo, uno de los primeros instrumentos que se introdujeron fueron las "Zonas Específicas para el Desarrollo Controlado" (ZEDEC, desde 1997 convertidos en Programas Parciales de Desarrollo Urbano), fundadas en 1987, tiempo en que se comenzaron a preparar las negociaciones para un acuerdo de libre comercio con Estados Unidos. Centrándose en una escala geográfica inferíor a las Delegaciones que implicó menos reglas que los "planes municipales", las ZEDECs fueron diseñadas inicialmente para controlar el uso de suelo en zonas donde la presión para convertir el uso de la tierra de vivienda a comercial era alta (por ejemplo en la zona de Polanco). Sin embargo, las ZEDECs también permitieron una planificación más detallada. Constituyeron "un instrumento de planeación de usos del suelo ad-hoc", como dijo Jorge Gamboa de Buen (2006: 5, propio énfasis), quien se desempeñó como jefe del Departamento de Planificación de la ciudad cuando Camocho Solís fue regente (1988-1994) y que hoy dirige Grupo Danhos. De esta manera, las ZEDECs rápidamente se convirtieron en un instrumento de desarrollo de la gran propiedad. Fueron esenciales en el desarrollo del nuevo CBD en Santa Fe desde finales de los ochenta, ya que permitieron cambiar el uso del suelo en la antigua mina de arena y luego basurero, lo que abrió la puerta para la construcción de oficinas, viviendas de lujo y centros comerciales.

Gamboa de Buen es claro tanto en el uso que se ha hecho de los ZEDECs como en la relación con la creciente globalización de la economía mexicana en este tiempo. Según él, con la introducción de los ZEDECs se ha "logrado, por primera vez en la historia de la ciudad, ajustar los precios al potencial de desarrollo" (Gamboa de Buen, 1994: 130). Explica, en otro lugar, cómo el ZEDEC Santa Fe ha sido instrumental para la construcción del nuevo CBD: "(E)mpezaron a aparecer estos cuates (como Salinas de Gortari, Camacho Solís, y otros) y con el libre comercio, empezaron a darse cuenta que el mercado también demandaba buenos proyectos. Santa Fe venía como anillo de dedo, era un terreno del Gobierno del Distrito Federal, estaba en el poniente, en donde el desarrollo tradicionalmente quería ir... De repente nos dimos cuenta que teníamos un terreno que había cumplido con ciertos elementos y tenía potencial. Por lo tanto decidimos hacer un ZEDEC... Hicimos Santa Fe Santa Fe le dio mucha confianza a la ciudad, generó mucho dinero, nuevos esquemas de desarrollo inmobiliarío e hizo que esta nueva generación de desarrolladores inmobiliaríos crecieran bien" (Gamboa de Buen, citado en Márquez López, 2012: 281-3).

Un siguiente paso para aumentar la libertad de intervenciones urbanas fue tomado en la regencia de Camacho Solís, cuando se introdujo el "Sistema de Transferencia del Potencial de Desarrollo Urbano". Este instru- 
mento permitió ceder el derecho de construir a un cierto nivel de una zona (básicamente el Centro Histórico) a otra, siempre y cuando se pagara una indemnización y la zona que lo recibiera fuera asignada como un área con potencial para el desarrollo (económico) o la integración metropolitana. Mientras que el propósito original del instrumento era generar medios para la conservación de los edificios en el Centro Histórico y densificar las partes de la ciudad con potencial de crecimiento, se ha utilizado el "Sistema de Transferencia del Potencial de Desarrollo Urbano" con frecuencia en la remodelación de Paseo de la Reforma: "La aplicación de esta norma, jugó un papel fundamental para iniciar la proliferación de magnos edificios en los predios con frente al Paseo de la Reforma, y al mismo tiempo significaba un buen esquema de inversión para los desarrolladores, en la medida en que podían incrementar la intensidad de la construcción en sus predios o inmuebles" (Márquez López, 2012: 158). La primera aplicación del instrumento fue en la Torre Mayor con sus 55 pisos. A pesar del retraso en la construcción, el arrendamiento de este edificio fue muy exitoso, por lo cual la Torre Mayor está discursivamente construida como un caso modelo para las ventajas de la planificación urbana flexible que es necesarío para la construcción física de la ciudad global. Por ejemplo, en una conferencia impartida a la Asociación Mexicana de Arquitectos y Consultores, Arturo Aispuro (2007: 18), el entonces jefe de la SEDUVI, que antes y después trabajó para Reichmann International, desarrolladora de la Torre Mayor, acompañó su promesa de más "facilidades administrativas" y de la "simplificación de los procedimientos para la autorización del proyecto" con una diapositiva de la Torre Mayor. De hecho, de los proyectos actualmente en marcha en Reforma, por lo menos diez superarán el límite de altura de 25 pisos establecidos por el Plan Maestro.

Un segundo objetivo de la reforma de la planificación urbana ha sido una especificación espacial más detallada de las áreas para la planificación urbana. Mientras que los ZEDECs ya han sido importantes en ese sentido, porque permitieron intervenciones en sitios bien definidos, los gobiernos de la ciudad de López Obrador (2000-2005) y Ebrard Casaubón (2006-2012) mejoraron aún más las oportunidades para la planificación específica mediante el establecimiento de "Corredores de Inversión y Desarrollo" y "Corredores de Integración y Desarrollo", respectivamente (CIDs). Según el gobierno de la ciudad, en los CIDs, pero particularmente a lo largo de Paseo de la Reforma, el desarrollo económico debe ser estimulado, y por eso las intervenciones urbanas deberían centrarse principalmente en ellos. Por lo tanto, la orientación geográfica de la planificación cambió escalas, de la planificación "para todos" (el conjunto de la ciudad) a la "planificación para las excepciones", los Ilamados proyectos estratégicos (para la estrategia de revalorización del suelo a través de megaproyectos en la Ciudad de México, ver Olivera y Delgadillo, 2014). Este cambio había facilitado, según SEDUVI, mecanismos ágiles de planificación, regulación y gestión, y permitió la acción concertada y eficaz (Aispuro, 2007). Hacer las intervenciones urbanas espacialmente más específicas y delimitadas se ha vuelto obligatorío porque el gobierno de la ciudad comenzó a depender de la colaboración públicoprivada. A lo largo de Reforma, desde el año 2001 la inversión pública "ha tenido como objetivo mejorar las condiciones de las inversiones inmobiliarias" (Romero Castillo, 2011: 62). Por otra parte, Ebrard Casaubón estableció incentivos financieros directos y estímulos fiscales para atraer a los desarrolladores las CIDs y, en particular, para Reforma, donde los beneficios fiscales equivalían unos 460 millones MXN entre 2002 y 2009 (Márquez López, 2012). Víctor Lachica, director general de la empresa de bienes raíces Cushman \& Wakefield, resumió que el apoyo del gobierno capitalino seguramente ha aumentado el valor de la zona de Reforma y estimuló la inversión en ella (El Universal, 2008).

El tercer tema de las reformas ha sido la centralización de la toma de decisiones. A finales de la década de 1980, el Departamento de Planificación de la ciudad tenía todo el poder para imponer sus proyectos (por ejemplo, Santa Fe) "porque no lo compartía (el poder) con la Asamblea (Legislativa del Distrito Federal), con los delegaciones o secretarías" (Gamboa de Buen, citado en Márquez López, 2012: 278). Cuando el PRD ganó las primeras elecciones democráticas en 1997, los procesos de democratización y descentralización se pusieron en marcha. Sin embargo, a más 
tardar con el gobierno de Ebrard Casaubón, comenzó una recentralización de la toma de decisiones, devolviendo la autoridad para aprobar los proyectos de desarrollo a la SEDUVI (GDF, 2008). Esto facilitó la política de estímulos fiscales para el sector inmobiliarío, a través de la simplificación de las normas (por ejemplo el principio de "one window shopping" para las licencias de proyecto de construcción o renovación) y su flexibilización (por ejemplo que las decisiones sobre el uso de la tierra o en las alturas del edificio deben ser manejadas complacientemente). Un paso significativo hacia la centralización de la toma de decisiones ha sido la aprobación de la nueva "Ley de Desarrollo Urbano" en 2010, que institucionalizó el papel central de la SEDUVI. Los nuevos decretos de ley plantean que el Parlamento de la ciudad tiene 60 días hábiles para aprobar lo propuesto por el alcalde o la autoridad de planeamiento. $\mathrm{Si}$ este período pasa sin comentarios, la propuesta se considera aprobada. En segundo lugar, las Ilamadas "Áreas de Gestión Estratégica" fueron introducidas, y se definen como zonas o corredores urbanos clave de importancia económica. Allí, los cambios del uso de la tierra y/o intervenciones en el entorno construido se hacen posibles sin la consulta obligatoria de los vecinos y la aprobación en la Asamblea Legislativa del Distrito Federal. Con respecto a las "Áreas de Gestión Estratégica" las decisiones son tomadas por el "Comité Técnico de Modificaciones a los Programas de Desarrollo Urbano", que está compuesto por cinco representantes del gobierno de la ciudad (entre otros los jefes de SEDUVI y el Departamento de desarrollo económico), nueve diputados, la cabeza del municipio respectivo y cuatro representantes de los vecinos, que tiene voz pero no voto.

\section{Planificación y las "necesidades" de mercado inmobiliario}

En suma, desde la década de 1980 las reformas introdujeron instrumentos de planificación urbana que permitieron un manejo más rápido, más flexible y específico de lugar de proyectos de construcción. Las políticas urbanas son caracterizadas por un papel proactivo del gobierno de la ciudad que uti- liza directa (por ejemplo estímulos fiscales) e indirectamente (por ejemplo, la inversión en infraestructura) incentivos para atraer a los inversionistas en el desarrollo inmobiliarío. Teniendo en cuenta estas príoridades, se puede concluir que los gobiernos del Distrito Federal siguieron lo que Burgess y Carmona (2009: 12) Ilaman un cambio universal hacia la planificación estratégica urbana. Esta se basa, con su enfoque en solo aqueIlos "aspectos o áreas que son estratégicos o importantes" (UN-HABITAT, 2009: xxiv; traducción del autor), en estrategias proactivas y adaptables, ni apuntando la ciudad como un todo ni sus distritos, pero de forma flexible crea entidades espaciales que son definidas por su potencial económico. Por otra parte, la planificación estratégica urbana es universal no solo porque se despliega en ciudades alrededor del mundo, sino también porque se ha ido desarrollado este enfoque transnacionalmente, dentro de las redes transfronterizas de ciudades y organizaciones internacionales como la ONU-Habitat (Parnreiter, 2011).

Como sugiere la literatura emergente sobre los flujos transnacionales de las ideas y prácticas de planificación (por ejemplo, Healey, 2012; Faulconbridge \& Grubbauer, 2015), en la llegada de esta norma mundial a la Ciudad de México "las movilidades de política urbana y los circuitos globales de conocimiento" (McCann, 2011, traducción del autor) jugaron un papel importante. La agenda de la ONU-Habitat, por ejemplo, es claramente "diseñado para influir en la planificación en la región" (América Latina y el Caribe; Irazábal, 2009: 57, traducción del autor). En México, sin embargo, el impacto de esta organización es evaluado diversamente. Mientras que a nivel nacional sus propuestas de políticas son, según varíos arquitectos y urbanistas entrevistados en la Ciudad de México, influenciadas por la ONU-Habitat, en las ciudades hasta ahora no se ha desarrollado ningún proyecto de cooperación técnica entre ONU-Habitat y el gobierno.

Sin embargo, la movilidad de las políticas urbanas ha sido esencial para los cambios en la planificación urbana en la Ciudad de México. Los expertos encuestados acuerdan que las ideas se copiaron principalmente de Francia (desde donde, por ejemplo, el "sistema de transferencia del potencial de 
desarrollo urbano" ha sido importado, aunque este instrumento fue creado por primera vez en los Estados Unidos) y luego principalmente de España. En particular, la idea de "la planificación a través de proyectos" (Burgess \& Carmona, 2009) se puso en práctica en Barcelona, Bilbao y Zaragoza ha sido influyente, y se trajo a México por las empresas españolas $y$, sobre todo, por Jordi Borja Urban Technology Consulting S.L. Borja, quien desempeñó un papel crítico en la transformación de Barcelona desde finales de los ochenta, fomentó la puesta en marcha de planes estratégicos en la Ciudad de México y otras ciudades de América Latina (González, 2011). "Borja viene cada año", dice un entrevistado, arquitecto de la Universidad Nacional Autónoma de México (UNAM), "y las empresas españolas de planificación, todos ellos están aquí'. En una vena similar, un ex jefe de la SEDUVI afirma en la entrevista que "Jordi viene con frecuencia, y es un buen amigo de muchos de nosotros". Los encuestados también indican que España es, debido a la lengua compartida, un destino preferido para los estudiantes mexicanos de arquitectura, urbanismo y geografía, que estudian en el extranjero, lo que hace que los ejemplos españoles se vean filtrados en la formación local.

Estudiando en el extranjero también cambia las actitudes de los estudiantes pertenecientes a la élite urbana $y$, en particular, a su fracción inmobiliaria. Gamboa de Buen, por ejemplo, recuerda que a lo largo de los ochenta "de repente apareció una generación de cuates muy jóvenes... que habían estudiado fuera, algunos venían de familias inmobiliarias, otros simplemente habían estudiado finanzas. De repente les atrajo lo que estaba pasando en Londres, Nueva York: en el mundo había un boom de regeneración de ciudades... (Nos dimos cuenta que la regeneración) no solo es un tema cuestión de dinero, es cultura e idiosincrasia, es un tema de ponerse las pilas" (Gamboa de Buen, citado en Márquez López, 2012: 280f). Esta autoconciencia creciente de la comunidad de bienes raíces, su entusiasmo para la acción, conduce a la cuestión de las relaciones entre el gobierno urbano y la economía inmobiliaria, que es decisiva para responder a la pregunta de cómo los cambios en la planificación ur- bana y la gestión en la Ciudad de México se han logrado.

Todos los encuestados están de acuerdo sobre la fuerte influencia que las empresas inmobiliarias han tenido en la modificación de las leyes y prácticas de planificación urbana, independientemente del partido político en cargo (hasta 1997 el PRI y el PRD desde entonces). Un ex funcionarío de la SEDUVI subraya en la entrevista que "los grandes canales son las relaciones que yo diría parecen ser informales entre los gobernadores (de la ciudad) y los sectores empresariales, los desayunos de trabajo famosos que son habituales, etc. Y también las relaciones que se desarroIlan en los eventos oficiales a los que están invitados los empresaríos. Estos son grandes elementos de transmisión de información". Es importante señalar que el entrevistado habla de relaciones que "'parecen' ser informales". En realidad, son estructurales y eso no solo en un análisis teórico de "la urbanización del capital" (Harvey, 1985), sino también en su lado práctico. Un número de personas muy influyentes en la Ciudad de México ha desarrollado sus carreras con una pierna en las políticas urbanas y la otra en negocios inmobiliaríos. Cuando Camocho Solís dejó el cargo como regente en 1994, muchas de las personas que lo rodeaban inmediatamente se convirtieron en el sector inmobiliarío. Destacan Gamboa de Buen y Arturo Aispuro, que ya se ha mencionado como han sido instrumental en el desarrollo de Santa Fe y la Torre Mayor. La carrera de este último particularmente se caracteriza por cambios de balancín de la autoridad de planeamiento de la ciudad a la industria de bienes raíces y viceversa. Aispuro trabajó en los tiempos cuando Gamboa de Buen dirigió la SEDUVI como su Director General, para cambiarse a Reichmann International en 1994, donde llegó a ser Vicepresidente de Desarrollo. Aprovechando de esta situación, Aispuro fue, según Gamboa de Buen (citado en Márquez López, 2012: 285), la persona que realmente "empujó la Torre Mayor". En 2006 Aispuro regresó a la SEDUVI como su cabeza. En esta función anunció que la Torre Diana, desarrollado también (entre otros) por su antigua compañía Reichmann International, contaría con todos los beneficios fiscales que el gobierno de la ciudad ofrecía a los proyectos de Reforma (El Universal, 2009), aunque la Torre 
Diana se encuentra a dos cuadras de distancia. Poco después del anuncio Aispuro dejó la SEDUVI para abrir su propia empresa de arquitectura y consultoría.

Ebrard Casaubón, jefe de gobierno de 2006 a 2012, desarrolló su vida política bajo el patrocinio de Camacho Solís y así fue como se vinculó íntimamente con este grupo y con el sector inmobiliarío, aunque nunca trabajó directamente en este. Cuando Ebrard Casaubón asumió el cargo en 2006, la relación entre el gobierno de la ciudad y el sector inmobiliarío se hizo más fuerte que nunca. Mientras que durante la regencia de López Obrador la influencia de las empresas de bienes raíces en la política urbana se desarrolló un poco "tímidamente", como un ex colaborador de la SEDUVI dice en la entrevista, impulsada también por el esfuerzo del gobernador para crear empleo y gravar los ingresos de la ciudad, "en el caso de Ebrard es diferente. En este caso, surgió una relación estructural con el sector inmobiliarío. ... La política de Ebrard abiertamente estaba inclinada hacia la capital inmobiliarío". Incluso la oposición derechista del PAN acusa al gobierno de Ebrard por haber caído de rodillas ante los intereses de la economía de bienes raíces (Villanueva, 2008).

Gamboa de Buen (citado en Márquez López, 2012: 284) describe cómo las relaciones se trabajaron en el caso de la Torre Mayor: "Un día me dice Camacho (Solís): oye has oído hablar de un señor que se llama Reichmann? Le dije que sí... (y) el presidente me dijo que Isaac Saba (empresarío mexicano, 1923-2008) se lo había presentado y entonces quiere venir a verme. Total Ilegó Paul Reichmann. Juan Enríquez Cabot (entonces director de Servimet, quien empujó el desarrollo de Santa Fe) y yo lo paseamos por toda la ciudad. ... y luego dijo (Reichmann) que quería hacer el edificio más alto de México sobre Reforma, pero le dijimos que ahí no había terrenos, y dijo: ¿ahí no hay terrenos? ¿Esa cosa que es? Se le ocurrió que en la entrada a Reforma debería haber dos torres. ¿Qué es esto? ¿Un cine? ¿De quién? Del gobierno. Lo compro, y ¿qué es eso de al lado? Una guardería de Seguro Social. Tenemos que conseguirla, ¿y del otro lado? Unos edificios viejos. Tenemos que tumbarlos. Al final... Io ayudamos a comprar el Cine Chapultepec, fue complicado. ... Y luego la guardería. ... Se ensambló la tierra, eso no había pasado antes en México, ahora ya somos expertos". Lo que suena caricatura en la entrevista es percibido por los arquitectos y planificadores entrevistados como fuertes presiones: "Ahí están los intereses (de los empresaríos de bienes raíces), insisten, hay esta solicitud de apoyo", dice un arquitecto que en varias ocasiones hizo auditorías ambientales para los proyectos de construcción. Otro participante, que ha trabajado en temas de planificación urbana y regional para el gobierno nacional y la ciudad y que también ha sido director ejecutivo en empresas de arquitectura del sector privado, afirma que "el sector inmobiliarío busca la máxima rentabilidad, y que la ley no limita la capacidad de la inversión". Y luego se pasa a describir las consecuencias para la política urbana: "Yo diría que en primer lugar que el sector inmobiliarío pensaba que los planes no tendrían que tener ningún efecto y tuvieron éxito es eso. Los planes se hacen, pero no se respetan. Por lo tanto, las presiones del sector inmobiliarío son muy eficaces". Preguntado sobre si los nuevos instrumentos mencionados anteríormente se han introducido cediendo a la presión del sector inmobiliarío, el entrevistado respondió: "Sí... Pero las cosas no han sido politizadas por los partidos políticos, lo que habría evitado que estén en la ley. Pero no, aprobado, sin esta politización, aprobada más bien como un instrumento útil, y ahora está allí en la ley".

Aunque otros entrevistados difieren en la medida en que reconocen que los instrumentos necesariamente no se han creado con el fin de servir al sector inmobiliarío, hay acuerdo en que bajo Ebrard Casaubón se utilizaron estos instrumentos a la "política de brazo absolutamente inclinado hacia el capital inmobiliarío", como el ya citado exoficial de la SEDUVI dice. También afirma que no se respetan los planes: "Los Programas Generales de Desarrollo Urbano tienen muy poca implicación que no son en realidad herramientas para la política. Los jefes de gobierno del Distrito Federal deciden lo que hacen en la ciudad, independientemente de lo que dice el proyecto, y entonces lo único que hacen es tratar de legalizar el programa una vez que se hacen las cosas". Un ex jefe de la SEDUVI coincide: 
"Yo diría que esto ha aumentado el apoyo casi ilimitado de lo que implica inversión en bienes raíces, prácticamente en cualquier lugar y a cualquier condición, ajustar los planos..., la Torre Mayor y todos los proyectos de Reforma no se discuten con el pueblo, en eso casos se discute con los inversores... La legalidad es una cosa muy flexible, se trata de interpretaciones, y el sector privado está dispuesto a hacer cualquier cosa para conseguir sus objetivos. Y claro, al final tienen su licencia, sí, pero muchas de estas cosas se han obtenido a través de la corrupción, por lo que es legal, pero es una legalidad muy discutible".

\section{Planificación estratégica y megaproyectos más allá de la Ciudad de México}

Como se mencionó anteríormente, el mercado de oficinas en las ciudades globales constituye el corazón del mercado inmobiliarío global (Fainstein, 2001; Lizieri \& Pain, 2014; Stevenson et al., 2014). Por eso, la planificación estratégica juega un papel clave en las políticas urbanas en estas ciudades (Thornley, 2000; Acuto, 2013). Sin embargo, eso no implica que esté reducida a las ciudades más importantes del mundo. Lungo Díaz (2005), por ejemplo observa la tendencia generalizada de que con la introducción del enfoque de la planificación estratégica los grandes proyectos urbanos pasaron a ocupar una posición privilegiada como acciones claves para impulsar el desarrollo de las ciudades. Así, de los 30 estudios de caso de planificación estratégica compilado en Carmona (2009), 13 se refieren a ciudades de América Latina: Bogotá, Buenos Aires, Caracas, Córdoba, La Plata, Ciudad de México, Montevideo, Panamá, Río de Janeiro, Santiago de Chile, San Salvador, São Paulo, y Valparaíso. Del mismo modo, Steinberg (2005) analiza diez casos, a saber Córdoba, Rosarío y Buenos Aires en Argentina, Santiago de Chile, Santa Cruz de la Sierra y La Paz en Bolivia, Trujillo y Lima en Perú, Bogotá en Colombia, y La Habana en Cuba. Mientras es, debido a limitaciones de espacio, más allá del alcance de este artículo para ir en más detalles acerca de los desarrollos en estas ciudades, cabe subrayar que se pueden generalizar las conclusiones a las cuales llegué en la evaluación crítica de la planificación estratégica en la Ciudad de México. Como dice Ciccolella (2010) acerca de las experiencias que se han hecho en América Latina:

"La 'Planificación Estratégica', si bien es de origen progresista y promueve contenidos y modalidades de planificación participativa, termina volviéndose a menudo reaccionaria, elitista y excluyente, sino ineficaz. Lamentablemente hay más casos de asociación de la planificación estratégica con el 'marketing urbano y la gentrification' que con una genuina democratización de los usos del suelo urbano y el desarrollo urbano integral e integrador. En algunos casos, especialmente en el ámbito latinoamericano, la planificación estratégica ha fortalecido la posición del capital especulativo y ha permitido... una convergencia entre especuladores, funcionaríos y desarrolladores urbanos, al promover un tipo de gestión del espacio urbano que termina estando al servicio predominantemente de las elites".

\section{Conclusiones}

El propósito de este trabajo ha sido el de contribuir al proyecto de desvelar las relaciones entre las dinámicas temporales de la acumulación de capital y la producción de nuevos espacios urbanos (Harvey, 1985). Saliendo de la noción de Sassen (1991) que la globalización ha traído consigo una nueva forma de centralidad económica en las ciudades globales, y tomando la Ciudad de México como caso de estudio, he demostrado que esta nueva centralidad se materializa a) en ciertas zonas de la ciudad, b) en la construcción de nuevos espacios de centralidad, y c) en megaproyectos. Por otra parte, he sostenido que para la producción de estos nuevos centros de la ciudad las políticas urbanas y, en particular, los mecanismos de planificación se han modificado, con el fin de permitir un manejo más rápido, más flexible y más específico de lugar de los proyectos de construcción. El motor detrás de estos cambios ha sido el mercado de bienes raíces, cuyos intereses determinan las políticas urbanas en la Ciudad de México (Garza, 1999). Los medios 
para hacerlo fue la implementación global de instrumentos de la planificación estratégica. Como se ha demostrado para el caso de la Ciudad de México, entre los principales mecanismos que han inducido los cambios en la política urbana son los circuitos globales de conocimiento y las presiones locales en la economía de bienes raíces.

Una conclusión que se puede extraer de este trabajo es que existe una íntima relación entre la transnacionalización de los negocios, de la planificación urbana y de la arquitectura. Mientras las corporaciones que funcionan a escala global demandan en cualquier parte donde se instalan oficinas de alta calidad y otros espacios para llevar a cabo sus negocios globales, estos nuevos centros económicos se están produciendo cada vez más según las lógicas organizacionales que son en sí mismas transnacionales. La planificación urbana estratégica con su enfoque en las áreas consideradas económicamente importantes y con su compromiso con los grandes proyectos urbanos se ha convertido en un estándar transfronterizo de cómo planificar y desarrollar espacios de centralidad (Parnreiter, 2011).

En cuanto a la política urbana, el cambio en la planificación y los procesos de toma de decisiones, corresponden a la imposición del modelo neoliberal a la escala urbana. El estudio de caso de la Ciudad de México demostró que a pesar de tener un gobierno en cierta medida de izquierda y en favor de los pobres, la planificación ha sido ajustada a favor de los desarrolladores con el fin de rehacer la ciudad de acuerdo con un tipo específico de desarrollo económico. Que el proyecto político neoliberal ha sido fundamental en el cambio a la planificación estratégica es reconocido por ONU-HABITAT (2004, 181: 173f, traducción del autor), que anuncia indefectiblemente la planificación estratégica como un medio para construir mejores ciudades: "A medida que la globalización obliga a las ciudades para volver a imaginarse a sí mis-

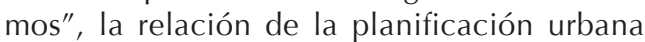
a las fuerzas del mercado tiene que ser redefinida, "removido de la planificación concebida como una limitación de las fuerzas del mercado (por ejemplo, por medio de leyes de zonificación) a un tipo de planificación empresarial que busca facilitar el desarrollo económico a través del mercado". En otros lugares UN-HABITAT (2009: $212 \mathrm{f}$, traducción del autor) dimite que "a no ser que el sistema de planificación puede ser visto para proporcionar un servicio eficiente y útil para el sector privado (...), siempre va a ser sometido a intentos de pasarlo por alto, subvertirlo o corromperlo". En su forma contemporánea dominante, la planificación estratégica, por lo tanto, no es un modo neutral, libre de contenido de la planificación. Por el contrarío, está incrustado en la "príoridad a la ventaja competitiva, la creación de paisajes urbanos atractivos, la búsqueda de la eficiencia y la productividad y la necesidad de reestructurar o desarrollar la ciudad en un contexto global", como Burgess \& Carmona (2009: 22, traducción del autor) concluyen su revisión de experiencias de planificación estratégicas en cuarenta y tres ciudades alrededor del mundo.

\section{Referencias bibliográficas}

AALBERS, M.B. (coordinador). Subprime Cities: The Political Economy of Mortgage Markets. Chichester: Wiley-Blackwell, 2012.

ACUTO, M. Global Cities, Governance and Diplomacy: The Urban Link. London: Routledge, 2013.

AISPURO CORONEL, A. Arquitectura y urbansimo sustentable. En: IV Congreso ADOC, AC. México D.F., 2007.

ARRIGHI, G. The Long Twentieth Century. Money, Power, and the Origins of Our Times. London: Verso, 1994.

BBVA RESEARCH. Situación Banca México. México D.F.: BBVA Research Mexico, 2012. Disponible en Internet:

http://www.bbvaresearch.com/KETD/fbin/ mult/100223_PresentacionesMexico_54_ tcm346-215427.pdf?ts=29112012

BRENNER, N. \& THEODORE, N. Cities and the Geographies of Actually Existing Neoliberalism. Antipode, 2002, Vol. 4, No 3, p. 349-379.

BURGESS, R. \& CARMONA, M. The shift of master planning to strategic planning. In: CARMONA, M. (editor). Planning through 
projects. Moving from master planning to strategic planning. 30 cities. Amsterdam: Techne Press, 2009, p. 12-42.

CARMONA, M. (editor). Planning through projects. Moving from master planning to strategic planning. 30 cities. Amsterdam: Techne Press, 2009.

CICCOLELLA, P. Metropolis y desarrollo urbano más allá de la globalización. Hacia una geografía crítica de la ciudad latinoamericana. Scripta Nova. Revista Electrónica de Geografía y Ciencias Sociales, 2010, Vol. XIV, No 331 (2). Disponible en Internet:

http://www.ub.edu/geocrit/sn/sn-331/sn331-2.htm

DUSSEL PETERS, E. Polarizing Mexico. The Impact of Liberalization Strategy. London: Boulder, 2000.

EXPANSIÓN. Las 500 empresas más importantes de México. México D.F.: CNN/ Expansión, varíos años.

FAINSTEIN, S., The city builders. Property development in New York and London. 19802000. Kansas: University Press of Kansas, 2001.

BRIDGE, J. \& GRUBBAUER, M. Introduction: Transnational building practices: actors, trajectories and modes of knowledge mobility. Global Networks, 2015, Vol. 15, № 3, p. 275-384.

GAMBOA DE BUEN, J. Ciudad de México. Una visión. México D.F., 1994.

GAMBOA DE BUEN, J. The Santa Fe node: Its beginnings. In: Urban Age Mexico City Conference, 2006. Disponible en Internet: http://downloads.Isecities.net/0_downloads/pdf_presentations/Mexico/D-1deBuen. pdf

GARZA, G. Global economy, metropolitan dynamics and urban policies in Mexico. Cities, 1999, Vol. 16, № 3, p. 149-170.

GOBIERNO DEL DISTRITO FEDERAL (GDF). Gaceta oficial No 248. México D.F., 2008.
GONZÁLEZ, S. Bilbao and Barcelona in motion. How urban regeneration models travel and mutate in the global flows of policy tourism. Urban Studies, 2011, Vol. 48, No 7, p. 1397-1418.

GRANT, R. \& NIJMAN, J., Globalization and the corporate geography of cities in the less-developed world. Annals of the Association of American Geographers, 2002, Vol. 92, № 2, p. 320-340.

GRUBBAUER, M. Architecture, economic imaginaries and urban politics: the office tower as socially classifying device. International Journal of Urban and Regional Research, 2014, No 381 , p. 336-359.

HAILA, A. Real Estate in Global Cities: Singapore and Hong Kong as Property States. Urban Studies, 2000, Vol. 37, № 12, p. 22412256.

HARVEY, D. The urbanization of capital. Oxford: Blackwell, 1985.

HARVEY, D. Globalization and the 'Spatial Fix'. Geographische Revue, 2001, Vol. 3, № 2, p. 23-30.

HEALEY, P. The universal and the contingent: Some reflections on the transnational flow of planning ideas and practices. Planning Theory, 2012, Vol. 11, № 2, p. 188-207.

INSTITUTO NACIONAL DE ESTADÍSTICAS Y GEOGRAFÍA (INEGI). Censos económicos 1999. Aguascalientes: INEGI, 1999.

INSTITUTO NACIONAL DE ESTADÍSTICAS Y GEOGRAFÍA (INEGI). Censos económicos 2009. Aguascalientes: INEGI, 2009.

IRAZÁBAL, C. Revisiting Urban Planning in Latin America and the Caribbean. Regional study prepared for Regional study prepared for Revisiting Urban Planning: Global Report on Human Settlements 2009. Disponible en Internet:

http://academiccommons.columbia. edu/download/fedora_content/download/ ac:130258/CONTENT/grhs_2009_regional. pdf 
JONES LANG LASALLE (JLL). Global real estate capital. Chicago: JLL, 2008.

JONES LANG LASALLE (JLL). Global Market Perspective Fourth Quarter 2012. Chicago: JLL, 2012a.

JONES LANG LASALLE (JLL). Global Capital Flows Database. Chicago/London/Singapore: JLL, $2012 \mathrm{~b}$.

KING, A. Spaces of global cultures. Architecture, urbanism, identity. London: Routledge, 2004.

KRÄTKE, S.; WILDNER,K. \& LANZ, S., (editors). Transnationalism and Urbanism. London: Routledge, 2012

LIZIERI, C. Global cities, office markets and capital flows. In: DERUDDER, B.; HOYLER, M.; TAYLOR, P. \& WITLOX, F. (editors). International Handbook of Globalization and World Cities. London: Edward Elgar, 2012, p. 162-176

LIZIERI, C. \& PAIN, K. International Office Investment in Global Cities: The Production of Financial Space and Systemic Risk. Regional Studies, 2014, Vol. 48, № 3, p. 439-455.

MÁRQUEZ LÓPEZ, L., Cambios en la estructura y formación de un corredor urbano terciarío Paseo de la Reforma, 1970-2007. México D.F.: Tesis a obtener el grado de Doctor en Urbanismo, Programa de Maestría y Doctorado en Urbanismo, Universidad Nacional Autónoma de México, 2012.

MCCANN, E. Urban policy mobilities and global circuits of knowledge. Toward a research agenda. Annals of the Association of American Geographers, 2011, Vol. 101, № 1 , p. 107-30.

OLIVERA, P. Neoliberalismo en la ciudad de México: polarización y gentrificación. En: HIDALGO, R. y JANOSCHKA, M. (editores). La ciudad neoliberal. Gentrificación y exclusión en Santiago de Chile, Buenos Aires, Ciudad de México y Madrid. Santiago de Chile: Instituto de Geografía, Pontificia Universidad Católica de Chile /Relaciones Internacionales, Universidad Autónoma de Madrid, 2014, p. 151-177.
OLIVERA, P. y DELGADILLO, V. Políticas empresarialistas en los procesos de gentrificación en la Ciudad de México. Revista de Geografía Norte Grande, 2014, No 58, p. 111-133.

PARNREITER, C. Global cities in Global Commodity Chains: exploring the role of Mexico City in the geography of global economic governance. Global Networks, Vol. 2010, No 101, p. 35-53.

PARNREITER, C. Commentary. Toward the making of a transnational urban policy? Journal of Planning Education and Research, 2011, Vol. 31, No 4, p. 416-422.

PARNREITER, C. 20 Jahre NAFTA. Mexikos gescheitertes upgrading in globalen Güterketten. Zeitschrift für Wirtschaftsgeographie, 2013, Vol. 57, № 4, p. 216-236.

PARNREITER, C. Las ciudades latinoamericanas en la economía mundial: la geografía de centralidad económica y sus transformaciones recientes. Economía UNAM, 2015, Vol. 12, No 35 , p. 3-22.

PRADILLA COBOS, E. Los territoríos del neoliberalismo en América Latina. compilación de ensayos. Tlalpan: Miguel Angel Porrua, 2009.

ROMERO CASTILLO, L. Políticas públicas en la conformación del corredor urbano Centro Histórico de la Ciudad de México-Santa Fe. Arq.urb, 2011, No 6, p. 46-72.

SASSEN, S. The global city. New York, London, Tokyo. Princeton: Princeton University Press, 1991.

SASSEN, S. The new centrality. The impacts of telematics and globalization. In: SAUNDERS, W. (editor). Reflections on architectural practices in the nineties. New York: Princeton Architectural Press, 1996, p. 206218.

SECRETARÍA DE ECONOMÍA. Dirección general de inversión extranjera. Estadística oficial de los flujos de IED hacia México. 2012. Disponible en Internet: http://www.economia. gob.mx/comunidad-negocios/inversion-ex- 
tranjera-directa/estadistica-oficial-de-ied-enmexico

SMITH, M.P. Transnational urbanism. Locating globalization. Blackwell: Malden, 2001.

STEVENSON, S.; AKIMOV, A.; HUTSON, E. \& KRYSTALOGIANNI, A. Concordance in Global Office Market Cycles, Regional Studies, 2014, vol. 48, № 3, p. 456-470.

STEINBERG, F. Strategic urban planning in Latin America: Experiences of building and managing the future. Habitat International, 2005, Vol. 29, № 1, p. 69-93.

TAYLOR, P.; NI, P.; DERUDDER, B.; HOYLER, M.; HUANG, J. \& WITLOX F. (editors). Global urban analysis. A survey of cities in globalization. London: Routledge, 2011.

TAYLOR, P.; DERUDDER, B.; HOYLER, M. \& NI, P. New regional geographies of the world as practised by leading advanced producer service firms in 2010. Transactions of the Institute of British Geographers, 2013, Vol. 38, No 3, p. 497-511.
THORNLEY, A. Strategic planning in the face of urban competition. In: SALET, W. \& FALUDI, A. (editors). The revival of strategic spatial planning. Amsterdam: Royal Netherlands Academy of Arts and Sciences, 2000, p. 39-51.

UNITED NATIONS HUMAN SETTLEMENTS PROGRAMME UN-HABITAT. The state of the world's cities 2004/2005. Globalization and urban culture. London: Earthscan, 2004.

UNITED NATIONS HUMAN SETTLEMENTS PROGRAMME UN-HABITAT. Planning sustainable cities. Global report on human settlements 2009. London, 2009.

VILlANUEVA, J. Acusa a Arturo Aispuro de arrodillarse ante inmobiliarias, 2008. Diposnivle en Internet: http://www.cronica.com. mx/nota.php?id_nota $=364402$

WARD, P. Mexico City. Chichester: Academy Press, 1998. 